Purdue University Purdue e-Pubs

2000

\title{
Passive Control of Discrete Frequency Tones Generated by Coupled Detuned Cascades
}

\author{
S. Sawyer \\ University of Akron \\ S. Fleeter \\ Purdue University
}

Follow this and additional works at: https://docs.lib.purdue.edu/icec

Sawyer, S. and Fleeter, S., "Passive Control of Discrete Frequency Tones Generated by Coupled Detuned Cascades" (2000). International Compressor Engineering Conference. Paper 1365.

https://docs.lib.purdue.edu/icec/1365

This document has been made available through Purdue e-Pubs, a service of the Purdue University Libraries. Please contact epubs@purdue.edu for additional information.

Complete proceedings may be acquired in print and on CD-ROM directly from the Ray W. Herrick Laboratories at https://engineering.purdue.edu/ Herrick/Events/orderlit.html 


\title{
PASSIVE CONTROL OF DISCRETE FREQUENCY TONES GENERATED BY COUPLED DETUNED CASCADES
}

\author{
Scott Sawyer \\ Department of Mechanical Engineering \\ University of Akron \\ Akron, Ohio 44325
}

\author{
Sanford Fleeter \\ School of Mechanical Engineering \\ Purdue University \\ West Lafayette, Indiana 47907
}

\begin{abstract}
Discrete-frequency tones generated by rotor-stator interactions are of particular concern in compressor design. In the annular inlet and exit ducts, the acoustic waves are generated at the multiples of rotor blade pass frequency. Classical theory considers an isolated flat-plate cascade of identical uniformly spaced airfoils. The current analysis extends this tuned isolated cascade theory to consider coupled aerodynamically detuned cascades where aerodynamic detuning is accomplished by changing the chord of alternate rotor blades and stator vanes. In a coupled cascade analysis, the configuration of the rotor influences the downstream acoustic response of the stator, and the stator configuration influences the upstream acoustic of the rotor. This coupled detuned cascade unsteady aerodynamic model is first applied to a baseline stage configuration. This baseline stage is then aerodynamically detuned. The nominal aerodynamically detuned stage configuration is then optimized, with the stage acoustic response decreased $5 \mathrm{~dB}$ upstream and $6 \mathrm{~dB}$ downstream at the design operating condition.
\end{abstract}

\section{NOMENCLATURE}

$\mathrm{C}_{\mathrm{A}} \quad$ chord length of Cascade A

$C_{B} \quad$ chord length of Cascade $B$

$C_{r} \quad$ ratio of chord length of Cascade $B$ to Cascade $A$

$k$ reduced frequency $k=\omega c / W$

n rotor harmonic

os chordwise offset of Cascade B relative to Cascade A

S circumferential spacing

$\mathrm{S}_{\mathrm{o}} \quad$ circumferential distance between adjacent Cascade $\mathrm{A}$ and Cascade $\mathrm{B}$ airfoils

$S_{r} \quad$ circumferential spacing ratio $S_{r}=S_{0} / S$

$\gamma \quad$ stagger angle

$\omega \quad$ excitation frequency $\omega=\mathrm{nN}_{\mathrm{B}} \Omega$

$\Omega \quad$ rotor shaft frequency

\section{INTRODUCTION}

The noise signature for a compressor stage comprised of a rotor and stator includes a broadband noise level with large spikes or tones at multiples of the blade passing frequency. These discrete-frequency tones are generated by periodic blade row unsteady aerodynamic interactions between adjacent airfoil rows. Namely, turbomachine airfoil rows are subject to spatially nonuniform inlet flow fields resulting from either potential or viscous wake interactions. Potential flow interactions result from variations in the pressure field associated with the airfoils of a given row and their effect on the airfoils of a neighboring row moving at a different rotational speed. This type of interaction is of concern when the axial spacing between neighboring airfoils rows is small orflow Mach numbers are high. Wake interactions result from the impingement of wakes shed by one or more upstream rows upon the flow through a downstream airfoil row and can persist over considerable axial distances.

Both potential flow and wake interactions result in the generation of acoustic waves that may propagate unattenuated and interact with other airfoil rows. In this way, the airfoil rows of the turbomachine are coupled, i.e., the response of an airfoil row acts as an excitation to neighboring airfoil rows. As a result, cascade coupling analyses for standard uniformly spaced thin airfoil cascades with constant chord, i.e. tuned cascades, have recently been developed. Hanson [1] studied mode trapping to explain stage acoustic response. Buffum [2] used similar techniques to determine the influence of coupling on the aeroelastic stability of a one and one-half stage compressor. Finally, Silkowski and Hall [3] used mode-coupling techniques to determine the influence of neighboring blade rows on the aerodynamic damping of a multistage turbomachine. 
Current noise control and reduction methods usually are a combination of turbomachinery noise source control and suppression. Source control is accomplished by increasing axial spacing between adjacent airfoil rows and by selecting blade and vane number combinations to produce "cut-off," whereby the highest-energy acoustic modes decay exponentially with distance along the ducting. Source suppression is achieved with acoustic liners in the inlet and exhaust ducts. However, there is a need for more source noise control by design, not liner suppression. As a result, progress noise reduction is dependent on innovative passive noise control techniques. Aerodynamic detuning is one such relatively new concept for passive noise control.

Aerodynamic detuning is defined as designed airfoil-to-airfoil differences of an airfoil row. Thus, aerodynamic detuning influences the airfoil-to-airfoil unsteady aerodynamics of the row. These differences affect the fundamental driving force of discrete-frequency noise generation, the unsteady airfoil surface pressures. Due to aerodynamic detuning, the airfoils do not respond in a classical traveling wave mode typical of a conventional uniformly spaced tuned airfoil row. Aerodynamic detuning is beneficial in aeroelastic problems of flutter and forced response. Analytical and computational studies of cascades operating in both incompressible [4,5] and compressible flow fields [6] have shown that aerodynamic detuning is beneficial to flutter stability.

In this paper a model is developed to analyze the unsteady aerodynamics of coupled aerodynamically detuned thin airfoil cascades in a compressible subsonic flow. The linearized two-dimensional continuity and momentum equations for inviscid isentropic compressible flow are solved using wave theory, a technique first utilized for a uniformly spaced airfoil cascade. This technique is extended herein to analyze aerodynamically detuned airfoil cascades made up of alternate nonuniformly spaced airfoils with different chord lengths, elastic axis locations and chordwise offset positions.

\section{DETUNED CASCADE UNSTEADY AERODYNAMICS}

An aerodynamically detuned cascade, Figure 1 , is the combination of two uniformly spaced cascades, denoted as Cascade $A$ and Cascade $B$. These tuned cascades have the same stagger angle $\gamma$ and circumferential spacing S. The coordinate system for Cascade A is $(x, y, z)$, with the Cascade B coordinate system $\left(x^{\prime}, y^{\prime}, z^{\prime}\right)$. The airfoils of Cascade $A$ may have different chord lengths than those of Cascade $B$, with the airfoil chords denoted by $C_{A}$ and $C_{B}$ and Cascade $A$ to $B$ airfoil chord ratio defined as $C_{r}=C_{A} / C_{B}$. Additional parameters that define the detuned cascade geometry include the following. The tangential distance between adjacent Cascade $A$ and $B$ airfoils in the detuned cascade is $S_{0}$. The Cascade $A$ to Cascade $B$ airfoil spacing ratio is $S_{r}=S_{d} / S$. The chordwise offset os is the distance in the $\mathrm{z}$ direction from the stagger line of Cascade $\mathrm{A}$ to the stagger line of Cascade $\mathrm{B}$.

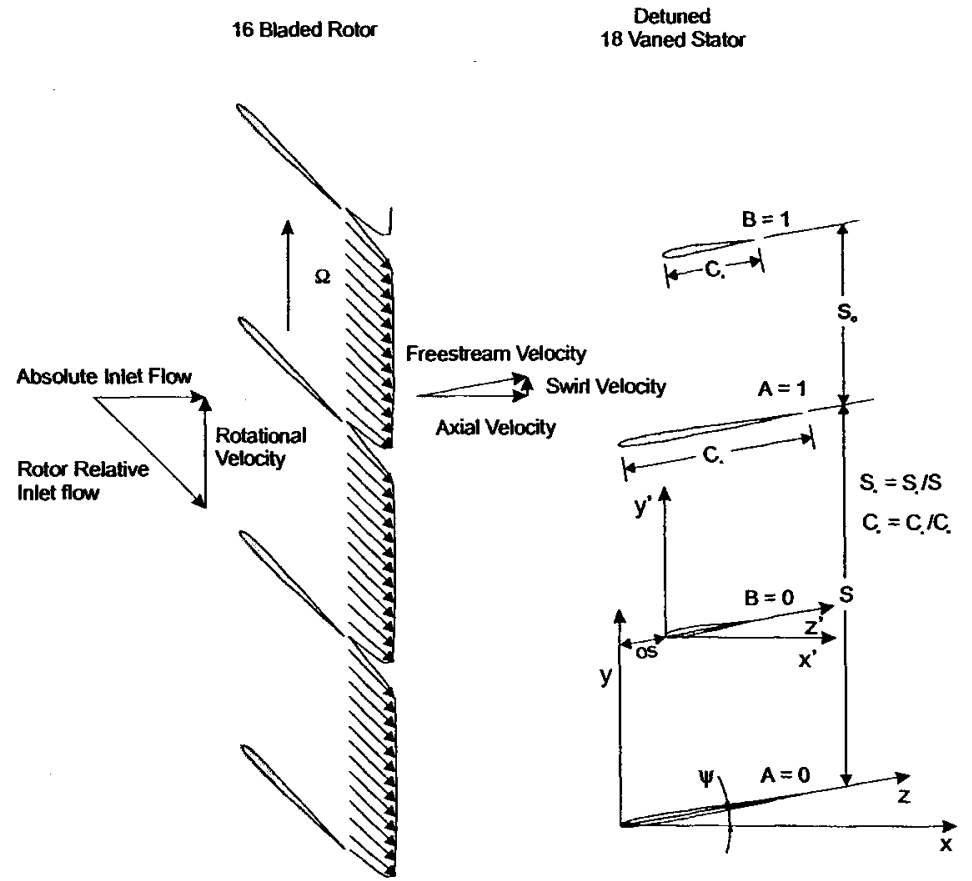

Figure 1. Detuned cascade geometry 


\section{PLANE WAVE SOLUTIONS OF THE LINEARIZED EULER EQUATIONS}

The plane wave solution of the two-dimensional inviscid compressible flow continuity and momentum equations linearized about a uniform mean flow are harmonic in time and periodic in $\mathrm{x}$ and $\mathrm{y}$.

$$
\left[\begin{array}{l}
u \\
v \\
p
\end{array}\right]=\left[\begin{array}{c}
\vec{u} \\
\bar{v} \\
\bar{p}
\end{array}\right] e^{i(\omega t+\alpha x+\beta y)}
$$

where $\alpha$ and $\beta$ are the axial and tangential wave numbers, and $\omega$ is the frequency.

Two families of solutions exist. The first solution family describes a vorticity wave that is simply convected with the mean flow, and has no associated pressure perturbation. The axial wave number for this vorticity wave solution is $\alpha_{3}=-\frac{\omega+V \beta}{U}$, with vortical gust solutions given the subscript 3 . The second solution family describes a pair of upstream and downstream going irrotational pressure or acoustic waves. The axial wave numbers for these pressure waves are $\alpha_{1,2}=\frac{U(\omega+V \beta) \pm a \sqrt{(\omega+V \beta)^{2}-\left(a^{2}-U^{2}\right) \beta^{2}}}{a^{2}-U^{2}}$ where the subscripts 1 and 2 are used to describe upstream and downstream going waves, respectively.

The propagation of the unsteady pressure perturbations or acoustic waves are described by the axial wave number $\alpha$ and depend on the values of the arguments under the radical. For $(\omega+V \beta)^{2}-\left(a^{2}-U^{2}\right) \beta^{2}>0$, two real wave numbers exist that describe a pair of acoustic waves that propagate away from the cascade unattenuated. For $(\omega+V \beta)^{2}-\left(a^{2}-U^{2}\right) \beta^{2}<0$, complex wave numbers describe acoustic waves that decay exponentially away from the cascade. If $(\omega+V \beta)^{2}-\left(a^{2}-U^{2}\right) \beta^{2}=0$, the cascade is in an acoustic resonance condition.

The airfoil cascade unsteady aerodynamic loading is modeled by replacing the airfoils with bound vortex sheets. The bound vortex distribution is then expanded in a Fourier series in the tangential direction, with the harmonics specified by the cascade mode index $v$. Unsteady cascade periodicity requirements then specify the tangential wave number $\beta$. It is equivalent to the spatial mode order $k_{\square}$ commonly used in annular duct acoustics. Substituting expressions for the interblade phase angle and pitch spacing and simplifying gives

$$
\beta=-\frac{1}{R}\left(n N_{\mathrm{B}}+v N_{V}\right), \quad v=0, \pm 1, \pm 2, \cdots
$$

where $\mathrm{R}$ is the radius and the term in brackets [7] is $k_{\theta}=n N_{\mathrm{B}}+v N_{v}$ with $\mathrm{k}_{\theta}$ the spatial mode order representing the number of lobes of the rotating pressure pattern.

\section{FAR-FIELD ACOUSTIC RESPONSE}

The unknown vortex distributions $\Gamma$ on Cascades $A$ and $B$ are found by solving the upwash integral equation in a manner analogous to the classical tuned cascade solution. The integral is evaluated numerically using the trapezoidal rule, and a variable transformation is used to resolve the high gradients near the leading edge. This yields a linear system of equations with the upwash specified and the vortex strength unknown. A polynomial curve fit that implicitly satisfies the Kutta condition is determined to approximate the vortex strength

The vortex curve fit coefficients $\delta$ are determined by solution of the linear system of equations.

$$
\left[\begin{array}{cc}
{\left[W_{A}\right]} & 0 \\
0 & {\left[W_{B}\right]}
\end{array}\right]=\left[\begin{array}{ll}
{\left[C_{A}\right]} & {\left[C_{B A}\right]} \\
{\left[C_{A B}\right]} & {\left[C_{B B}\right]}
\end{array}\right]\left[\begin{array}{ll}
{\left[\delta_{A}\right]} & {\left[\delta_{B A}\right]} \\
{\left[\delta_{A B}\right]} & {\left[\delta_{B B}\right]}
\end{array}\right]
$$

where $\mathrm{W}_{\mathrm{A}}$ and $\mathrm{W}_{\mathrm{B}}$ are the specified upwash distributions, $\mathrm{C}_{\mathrm{AA}}, \mathrm{C}_{\mathrm{BA}}, \mathrm{C}_{\mathrm{AB}}$ and $\mathrm{C}_{\mathrm{BB}}$ are the components of the coefficient matrix, and $\delta_{\mathrm{AA}}, \delta_{\mathrm{BA}}, \delta_{\mathrm{AB}}$ and $\delta_{\mathrm{BB}}$ are the vortex curve fit coefficients. 
To solve the upwash integral equation, the upwash is specified to satisfy the flow tangency condition on the airfoil surface. Two upwash conditions are considered: the convected vortical gust upwash and the acoustic wave upwash. With the $\delta$ vortex curve fit coefficients determined for the specified upwash condition, the vortex distributions are calculated and the acoustic response is given by

$$
P_{1}=-\frac{c}{S} v_{1} \int_{0}^{1} \Gamma(z) \exp \left\{-i\left(\alpha_{1} \cos \gamma+\beta \sin \gamma\right) z\right\} d z \quad P_{2}=-\frac{c}{S} v_{2} \int_{0}^{1} \Gamma(z) \exp \left\{i\left[1-\left(\alpha_{2} \cos \gamma+\beta \sin \gamma\right) z\right]\right\} d z
$$

where $P_{1}$ the upstream going acoustic wave is referenced to the cascade leading edge and $P_{2}$ the downstream going acoustic wave is referenced to the cascade trailing edge.

The combined acoustic response is determined by combining the individual upstream going components $\mathrm{P}_{\mathrm{AAl}}, \mathrm{P}_{\mathrm{BA1}}, \mathrm{P}_{\mathrm{AB} 1}$ and $\mathrm{P}_{\mathrm{BB} 1}$. The combined acoustic and shed wake response is calculated to account for the phase shift in the upwash and response from Cascade A to Cascade B.

\section{COUPLED CASCADE THEORY}

The potential fields of upstream and downstream airfoil rows and the convected vortical gusts of upstream airfoil rows excite a turbomachine airfoil row. The unsteady velocity perturbation at the solid airfoil surface produces an unsteady airfoil surface pressure required to satisfy the flow tangency condition. The unsteady airfoil loading then couples with the duct to produce a convected vortical wake and upstream and downstream going acoustic waves. In this manner, disturbances from upstream and downstream airfoil rows are transmitted and reflected by adjacent blade rows. The transmitted and reflected waves are now additional disturbances that excite the adjacent airfoil rows including the original airfoil row. The reflected and transmitted waves are not considered in a traditional isolated airfoil row analysis and can have a profound influence on the unsteady response.

An upstream going acoustic wave $P_{1 S}(n, v)$ from the stator is characterized by a rotor harmonic $n$ and stator mode index $v$. The upstream going acoustic wave is composed of the steady stator field $P_{15 x}$ and upstream going pressure waves reflected from rotor downstream going acoustic wave $S_{12} P_{2 R}$ and the rotor convected vortical gust $S_{13} \xi_{3 R}$, where $S_{12}$ and $S_{13}$ are the scattering coefficients.

$$
P_{1 S}(n, v)=P_{1 S X}(n, v)+\sum_{v^{\prime}} S_{12}\left(n, v ; n, v^{\prime}\right) P_{2 R}\left(n, v^{\prime}\right)+\sum_{v^{\prime}} S_{13}\left(n, v ; n, v^{\prime}\right) \xi_{3 R}\left(n, v^{\prime}\right)
$$

The scattering coefficients represent the stator response to a unit amplitude disturbance. The set of convected vortical gust and upstream and downstream going acoustic waves from the rotor $\left(P_{1 R}, P_{2 R}\right.$ and $\left.\xi_{3 R}\right)$ are composed of the steady rotor field $\left(\mathrm{P}_{1 \mathrm{RX}}, \mathrm{P}_{2 \mathrm{RX}}\right.$ and $\left.\xi_{3 \mathrm{RX}}\right)$ and the scattered waves $\left(\mathrm{S}_{11} \mathrm{P}_{1 \mathrm{~S}}, \mathrm{~S}_{21} \mathrm{P}_{1 \mathrm{~S}}\right.$ and $\left.\mathrm{S}_{31} \mathrm{P}_{1 \mathrm{~S}}\right)$ created by the stator upstream going pressure wave, with the coupling equations written in matrix form as

$$
\left[\begin{array}{cccccc}
I & 0 & 0 & -S_{11} & 0 & 0 \\
0 & I & 0 & -S_{21} & 0 & 0 \\
0 & 0 & I & -S_{31} & 0 & 0 \\
0 & -S_{12} & -S_{13} & I & 0 & 0 \\
0 & -S_{22} & -S_{23} & 0 & I & 0 \\
0 & -S_{32} & -S_{33} & 0 & 0 & I
\end{array}\right]\left[\begin{array}{c}
P_{1 R} \\
P_{2 R} \\
\xi_{3 R} \\
P_{1 S} \\
P_{2 S} \\
\xi_{3 S}
\end{array}\right]=\left[\begin{array}{c}
P_{1 R X} \\
P_{2 R X} \\
\xi_{3 R X} \\
P_{1 S X} \\
P_{2 S X} \\
\xi_{3 S X}
\end{array}\right]
$$

where the source vector is prescribed and the solution is determined through standard matrix techniques.

\section{RESULTS}

A classical analysis considers a rotor and stator in isolation, i.e. the rotor has no effect on the acoustic response of the stator and visa versa. Using a coupled analysis, the endeavor is to reduce the stage overall acoustic response comprised of a rotor and stator row and quantify the influence of the detuned stage configuration.

The influence of aerodynamic detuning on the discrete-frequency acoustic response is determined by applying this model to a tuned stage with 18 rotor blades and 38 stator vanes. At blade pass frequency, this design is "cut-off" and no propagating acoustic waves are generated for subsonic rotor relative Mach numbers. The tuned 
stator row has a pitch spacing of one chord and zero stagger. The stage is defined by the rotor-stator chord ratio of 0.474 (the rotor and stator have a pitch spacing of 1 chord). For a machine with a radius of $3 \mathrm{ft}$ spinning at 2,820 $\mathrm{rpm}$, the reduced frequency at blade pass frequency is 6.0. The rotor and stator are staggered at $-63.6^{\circ}$ and $0^{\circ}$, respectively. An absolute Mach number of 0.4 gives a rotor relative Mach number of 0.9 . Propagation of acoustic waves begins at an absolute Mach number of 0.08 .

The coupled cascade theory does not provide the amplitude of the steady potential fields and the shed vortical wake of the rotor and the stator. The following model is utilized.

$$
\left[\begin{array}{l}
P_{1 X} \\
P_{2 X} \\
\xi_{3 X}
\end{array}\right]=\left[\begin{array}{l}
\bar{P}_{1 X} \\
\bar{P}_{2 X} \\
\bar{\xi}_{3 X}
\end{array}\right]\left(1+\exp \left\{i\left(2 S_{r}\right) \pi n\right\}\right)
$$

where $\overline{\mathrm{P}}_{1 \mathrm{X}}, \overline{\mathrm{P}}_{2 \mathrm{X}}$ and $\bar{\xi}_{3 \mathrm{X}}$ represent the complex amplitude of the steady disturbance.

Figure 2 shows the acoustic response upstream and downstream of the propagating modes $(4,2)$ and $(6,2)$ and the total response over a range of Mach numbers from 0.08 to 0.4 for this baseline tuned stage configuration. Upstream the amplitudes of the propagating waves are comparable, but downstream the $2 \times B P F$ tone dominates.
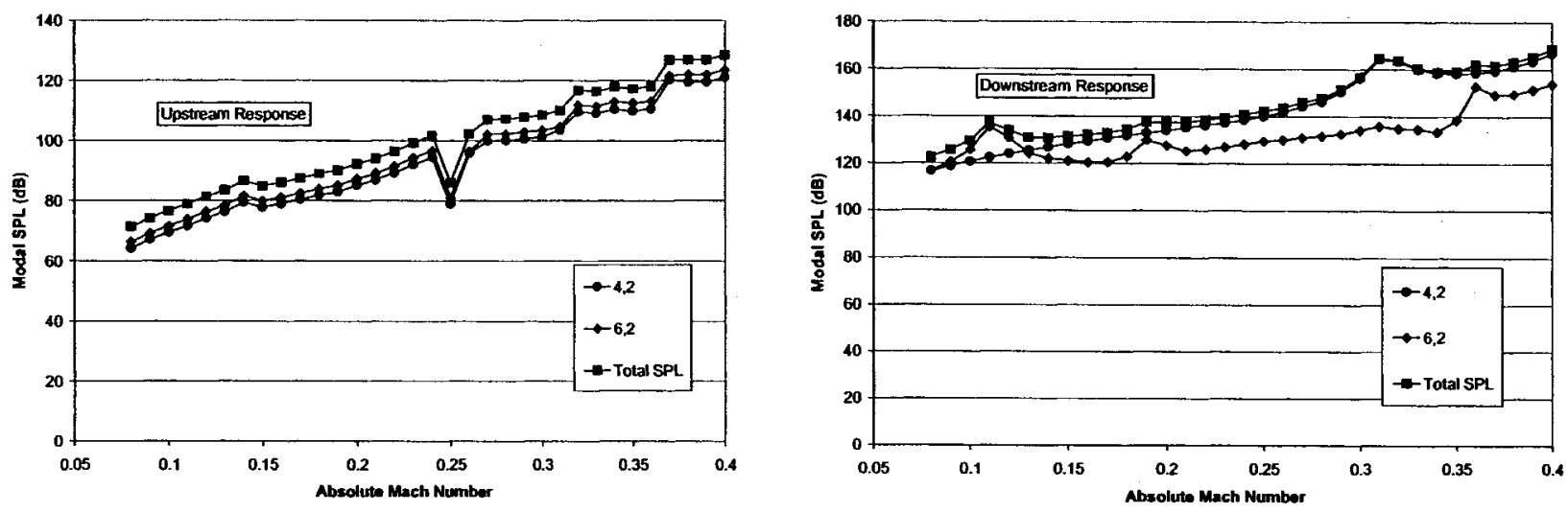

Figure 2. Coupled tuned cascade response

The baseline-detuned stage has rotor and stator chord ratios of 0.5 , rotor and stator spacing ratios of 0.5 and rotor and stator chordwise offsets of 0.0 . The half harmonic acoustic waves no longer cancel and there are many more nonzero propagating acoustic waves of lower amplitude, Figure 3.
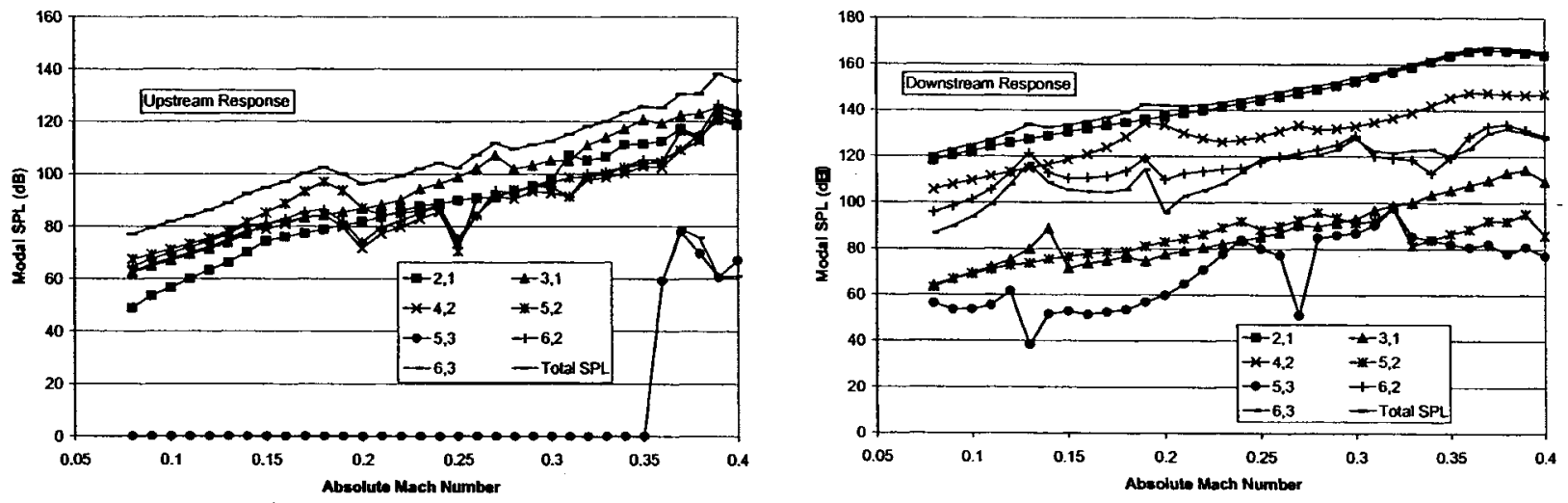

Figure 3. Coupled detuned cascade response

This arbitrary aerodynamically detuned configuration shows no advantage over the baseline-tuned stage in the acoustic response upstream and downstream of the stage, Figure 4. 

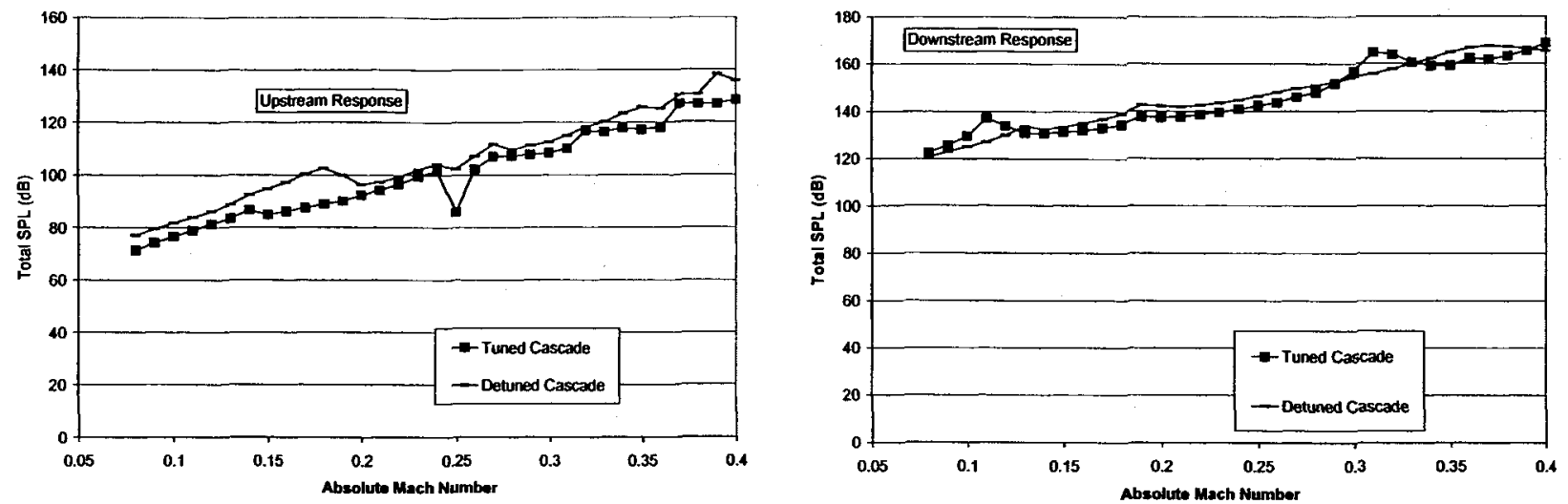

Figure 4. Comparison of tuned cascade and detuned cascade response

The question remains if an optimized detuned stage geometry can significantly lower the acoustic response. Thus, the chord ratio, spacing ratio and chordwise offset are varied individually at the 0.4 absolute Mach number condition to determine the optimum detuned stage configuration.

The influence of rotor and stator chord ratio is shown in Figure 5. The stage acoustic response is determined as a function of rotor chord ratio while the stator chord ratio is constant and a function of the stator chord ratio while the rotor chord ratio is held constant. One may initially suspect that a reduced splitter chord corresponds to reduced acoustic response, but this is not the case as the optimum stator chord ratio is 0.7 . The optimum rotor chord ratio is 0.3 as this condition minimizes the downstream acoustic response. The upstream acoustic response is a relatively weak function of the rotor chord ratio.
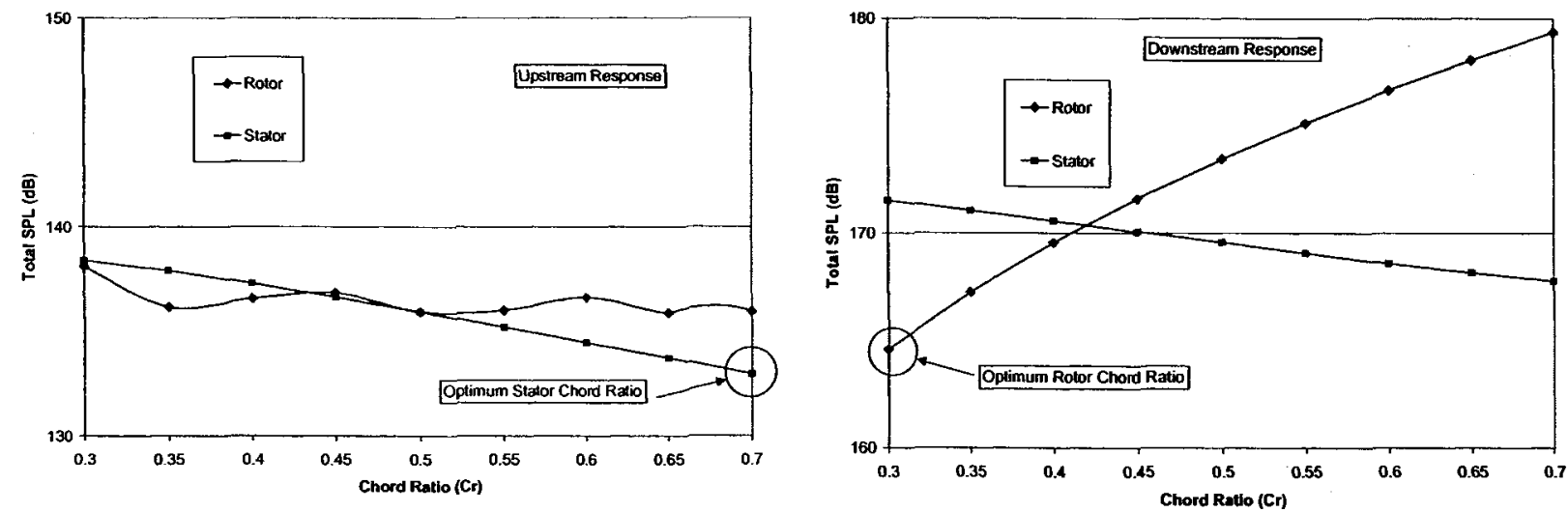

Figure 5. Effect of rotor and stator chord ratio on the coupled cascade response

There is no clear trend in the influence of the spacing ratio on the acoustic response of the stage. The optimum rotor and stator spacing ratios are 0.7 and 0.65 based on the minimization of the combined upstream and. downstream acoustic response.

Figure 6 shows the influence of the rotor and stator chordwise offset on the acoustic response of the stage. The stator chordwise offset has only a very small effect on the upstream acoustic response. Likewise, the rotor chordwise offset has a negligible effect on the downstream acoustic response. Thus, the optimum rotor and stator chordwise offsets are 0.35 for the rotor and 0.0 for the stator. 

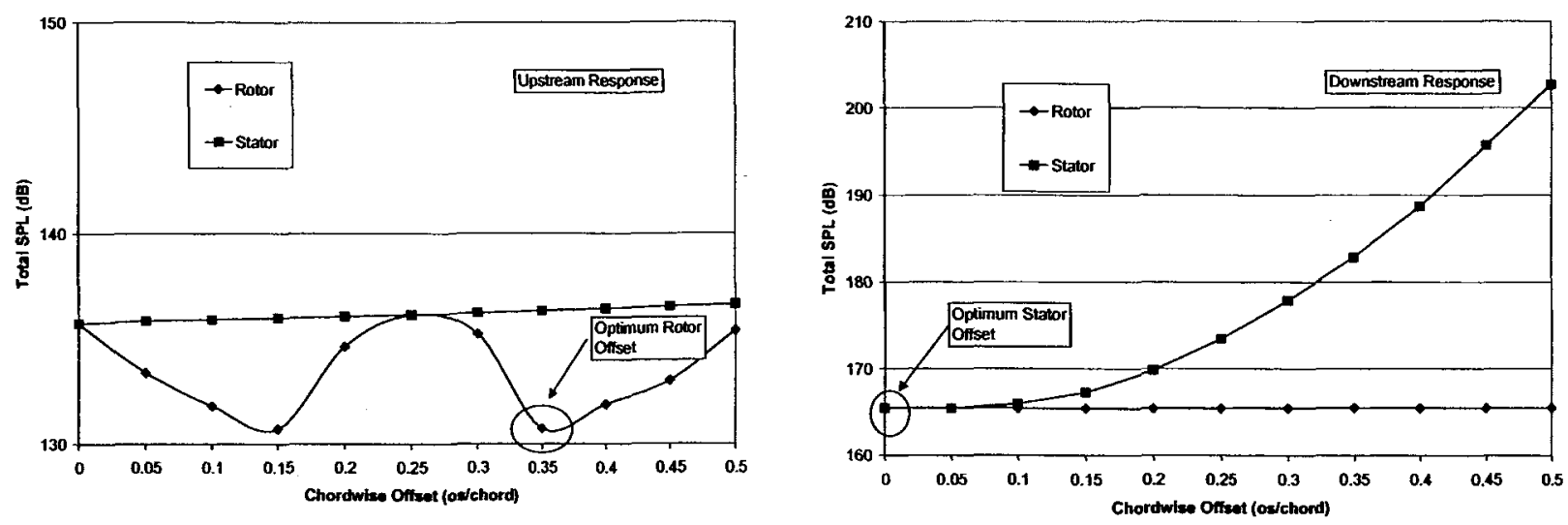

Figure 6. Effect of rotor and stator chordwise offset on the coupled cascade response.

The optimum detuned stage configuration for the 0.4 absolute Mach number operating condition has thus been determined. The rotor is configured with a chord ratio of 0.3 , a spacing ratio of 0.7 , and a chordwise offset of 0.35 . The stator configuration is 0.7 -chord ratio, 0.65 spacing ratio and no chordwise offset. Figure 7 shows that the optimized detuned stage configuration results in reductions of $5 \mathrm{~dB}$ upstream and $6 \mathrm{~dB}$ downstream as compared to the baseline stage at the 0.4 absolute Mach number condition.

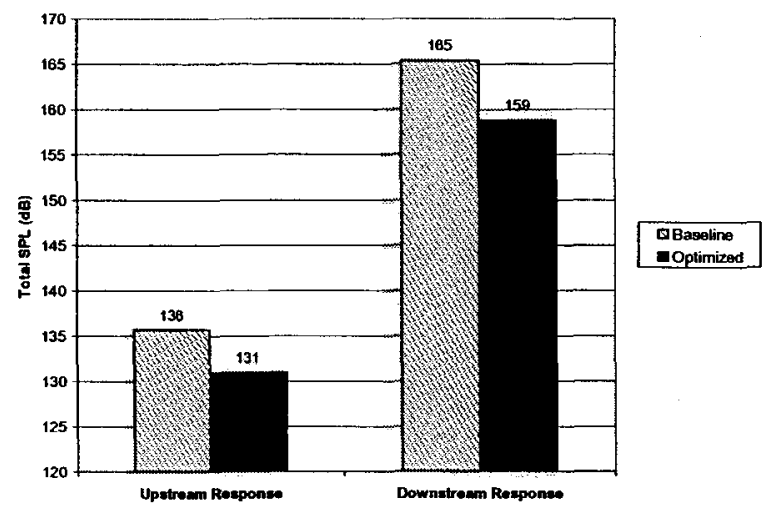

Figure 7. Combined acoustic response for the baseline and optimized detuned cascades at the $\mathrm{M}_{\mathrm{abs}}=0.4$

Figure 8, shows that there are noise reductions on the order of $5 \mathrm{~dB}$ upstream and downstream over the majority of the operating conditions.
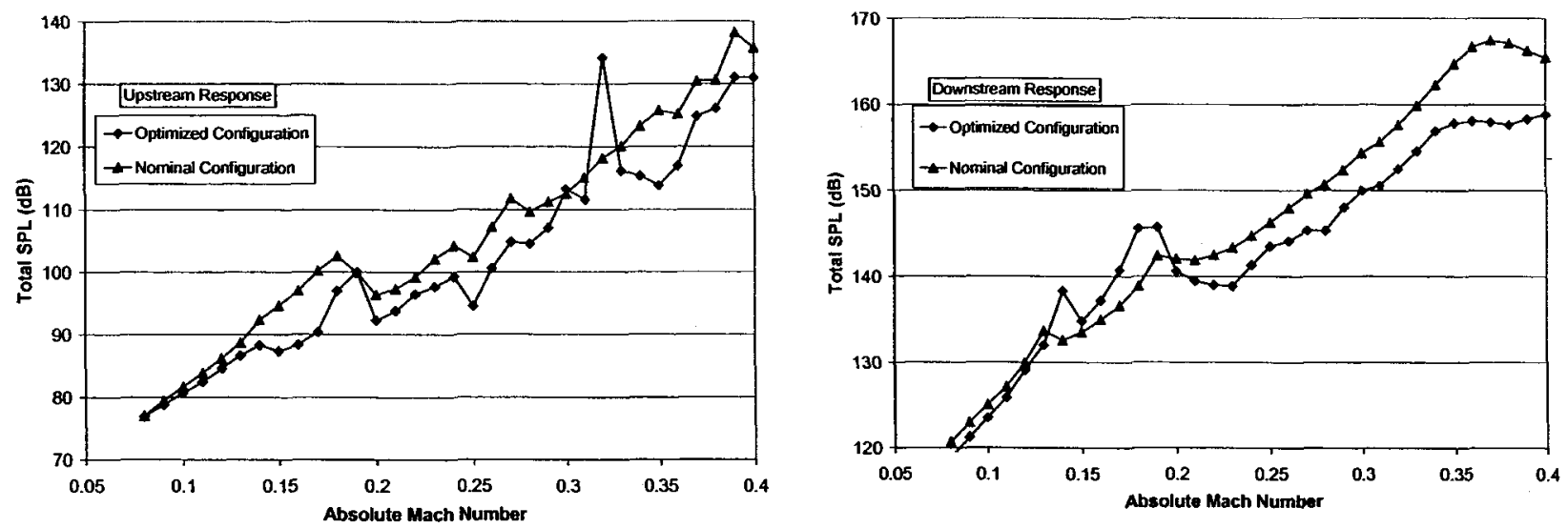

Figure 8. Comparison of optimized and nominal acoustic response of coupled detuned cascades 


\section{SUMMARY AND CONCLUSIONS}

An analytical model has been developed to determine the unsteady aerodynamics and subsequent acoustic response of aerodynamically detuned turbomachinery stage. The model considers compressible flow in two dimensions with small unsteady perturbations in pressure and velocity superimposed. The cascade model considers coupled, flat-plate uncambered airfoils at zero incidence with the mean flow. The rotor-stator coupling is accomplished through the scattering of acoustic and vorticity waves between the rotor and the stator.

The overall acoustic response of a baseline tuned stage configuration was determined over a range of operating conditions utilizing the coupled unsteady aerodynamic analysis. The acoustic response of the tuned stator row was compared to the response of the aerodynamically detuned stator. The optimum chord ratio, spacing ratio and chordwise offset were then determined for both the rotor and the stator at a single operating condition. This optimized stage configuration achieved noise reductions of 5 and $6 \mathrm{~dB}$ upstream and downstream at the design operating condition. The relative noise reduction was then determined over the range of operating conditions, with reductions on the order of $5 \mathrm{~dB}$ resulting over the majority of the operating conditions.

\section{ACKNOWLEDGEMENTS}

The NASA Glenn Research Center and the Air Force Office of Scientific Research sponsored this research. This support is most gratefully acknowledged.

\section{REFERENCES}

1. Hanson, D.B., "Mode Trapping in Coupled 2D Cascades - Acoustic and Aerodynamic Results," AIAA Paper 93-4417, October, 1993.

2. Buffum, D. H., "Blade Row Interaction Effects on Flutter and Forced Response," AIAA Paper 93-2084, 1993.

3. Silkowski, P. D. and Hall, K. C., "A Coupled Mode Analysis of Unsteady Multistage Flows in Turbomachinery," Transactions of the ASME, Vol. 120, July 1988, pp. 410-421.

4. Chiang, H.D., and Fleeter, S., "Passive Control of Flow Induced Vibrations by Splitter Blades," ASME Journal of Turbomachinery, Vol. 116, No. 3, July 1994, pp. 489-500.

5. Henderson, G.H. and Fleeter, S., "Oscillating Aerodynamics and Flutter of an Aerodynamically Detuned Cascade in an Incompressible Flow," International Journal of Turbo \& Jet Engines, Vol. 10, No. 4, 1993.

6. Sawyer, S. and Fleeter, S., "Flutter Stability of a Detuned Cascade in Subsonic Compressible Flow," AIAA Journal of Propulsion and Power, Vol.11, No. 5, September 1995.

7. S Tyler, J.M. and Sofrin, T.G., "Axial Flow Compressor Noise Studies," SAE Transactions, Vol. 70, 1962, pp. 309-332. 